\title{
BIOLOGIA REPRODUTIVA E POLINIZAÇÃO DE ESPÉCIES SIMPÁTRICAS DE DIPLUSODON (LYTHRACEAE)
}

\author{
Mariluza A. Granja e Barros \\ Herbário, Departamento de Botânica \\ Universidade de Brasília \\ Caixa Postal 4631 \\ 70919-900. Brasília, DF. Brasil
}

\begin{abstract}
RESUMEN
La biología reproductiva y la polinización de Diplusodon crulsianus Pohl, $D$. oblongus Pohl, $D$. ramosissimus Pohl, $D$. rosmarinifolius St.Hil, y $D$. villosus Pohl, fueron investigadas durante 1985, 1986 y 1991. Estas especies crecen simpatricamente en los "cerrados" del Distrito Federal (Brasil) y florecen sincrónicamente: $D$. oblongus con $D$. villosus y $D$. crulsianus con $D$. ramosissimus y $D$. rosmarinifolius. Diferencias en la morfología foliar, presencia o ausencia de pilosidad en las estructuras vegetativas y florales, y en el número de estambres, determinan la separación de las especies. Las flores de Diplusodon son productoras de polen, tienen antesis diurna, corola de color rosa fuerte, que contrasta con los estambres amarillos y muestran cierto grado de enantiostilia. Trigona spinipes L., Augochloropsis spp. y Apis mellifera L. se determinaron como polinizadores potenciales. Con relación a los sistemas reproductivos, las especies de Diplusodon son xenogámicas, ocurriendo autogamia facultativa en $D$. crulsianus y $D$. villosus ( $16 \%$ y $17 \%)$.
\end{abstract}

\section{ABSTRACT}

The reproductive biology and pollination of Diplusodon crulsianus Pohl, $D$. oblongus Pohl, $D$. ramosissimus Pohl, D. rosmarinifolius St. Hil, and D. villosus Pohl, were investigated during 1985, 1986 and 1991. These species occur sympatrically in "cerrados" (savanna-like vegetation) of the Federal District (Brazil), where they flower synchronously, D. oblongus with $D$. villosus (January to April) and $D$. crulsianus with $D$. ramosissimus and $D$. rosmarinifolius (May to August). Differences in leaf morphology, presence or absence of tomentum on the vegetative and floral structures and stamen number, separate the species. Diplusodon flowers have diurnal anthesis, flowers are magenta-colored contrasting with the yellow stamens and show some degree of enantiostyly. Trigona spinipes L., Augochloropsis spp. and Apis mellifera L. were considered potential pollinators. Diplusodon species are xenogamous with facultative autogamy in D. crulsianus and D. villosus (16\% and $17 \%)$.

\section{RESUMO}

A biologia reprodutiva e a polinização de $D$. crulsianus Pohl, $D$. oblongus Pohl, $D$. ramosissimus Pohl, D. rosmarinifolius St. Hil e D. villosus Pohl, foram investigadas durante 1985, 1986 e 1991. Estas espécies ocorrem simpatricamente em cerrados do Distrito Federal (Brasil) e florescem em sincronia: $D$. oblongus/ $D$. villosus (janeiro a abril) e $D$. crulsianus/ $D$. ramosissimus/ $D$. rosmarinifolius (maio a agosto). Diferenças na morfologia foliar, presença ou ausência de pilosidade nas estruturas vegetativas e florais, 
e no número de estames, determinam a separação das espécies. As flores de Diplusodon são produtoras de pólen, têm antese diurna, corola róseo-forte contrastando com estames amarelos e apresentam certo grau de enantiostilia. Trigona spinipes L., Augochloropsis spp. e Apis mellifera foram consideradas polinizadores potenciais. Com relação aos sistemas reprodutivos as espécies de Diplusodon são xenogâmicas, ocorrendo autogamia facultativa em D. crulsianus e D. villosus (16 \% e $17 \%$ ).

\section{INTRODUÇÃO}

Diplusodon Pohl é um gênero endêmico do Brasil, com cerca de 57 espécies (Graham et al., 1985; Lourteig, 1989). Ocorre particularmente em cerrados do CentroOeste, tendo sido também documentado em campos rupestres (Heringer et al., 1977; Ratter, 1980; Cavalcanti, 1995). As 5 espécies de Diplusodon aqui estudadas são freqüentes em cerrado (sensu lato), principalmente em campo cerrado e campo sujo (gradientes de cerrado) do Distrito Federal. Este é um estudo pioneiro sobre a biologia floral, polinização, simpatria e índice de enantiostilia em espécies do gênero Diplusodon, embora Oliveira (1991) tenha estudado o ciclo reprodutivo de $D$. orbicularis Koehne.

\section{MATERIAL E MÉTODOS}

O estudo foi desenvolvido na Fazenda Experimental da Universidade de Brasília - FAL/UnB (1555'S, 4755'W) durante os anos de 1985/1986, e dados complementares foram obtidos em 1991. As áreas de estudo foram selecionadas de acordo com a freqüência das espécies de Diplusodon. Foram monitorados 31 indivíduos de $D$. crulsianus, 78 de $D$. oblongus, 29 de $D$. ramosissimus; 39 de $D$. rosmarinifolius e 67 de $D$. villosus. Os representantes das espécies arbustivas $(D$. oblongus, $D$. ramosissimus e $D$. rosmarinifolius) ocorrem, em geral, formando agrupamentos, que variam de poucos a dezenas de metros e são provavelmente originados de um único xilopódio. Nas espécies subarbustivas ( $D$. crulsianus e $D$. villosus) os indivíduos ocorrem isolados. $D$. oblongus é a espécie mais frequente, sendo encontrada até no cerradão da $\mathrm{FAL}$. $D$. ramosissimus é a menos frequente, tendo sido observadas somente duas populações, ambas em campo cerrado. As outras espécies ocorrem principalmente em campo cerrado e campo sujo. Foram registrados dados referentes às características morfológicas, vegetativas e florais. Foi acompanhada a fenologia de floração e frutificação dos indivíduos de cada população, assim como, foram contados os botões florais, flores e frutos. Para a investigação dos sistemas reprodutivos, foram realizados 5 testes de polinização artificial (auto-polinização espontânea, auto-polinizaçâo induzida, apomixia, polinizaçâo interespecífica e polinização cruzada) sendo comparados com a polinização natural (controle). Os botões florais foram ensacados e as flores a serem tratadas foram emasculadas (retirada das anteras) durante a pré-antese para os testes de apomixia, de polinização cruzada e de polinização interespecífica. Foram usadas linhas coloridas para a identifição dos testes realizados. Após cada tratamento, as flores foram reensacadas. Para os testes de polinização interespecífica de espécies que florescem assincrônicamente, foi usado pólen estocado em tubo de plástico hermeticamente fechado, contendo no interior uma camada de cloreto de cálcio, uma camada de algodão, o pólen, e mantido em geladeira. Para o estudo do 
desenvolvimento dos tubos polínicos, pistilos pós-polinizados foram coletados $(24 \mathrm{~h}, 48 \mathrm{~h}$, $72 \mathrm{~h}$ após os tratamentos) e conservados em FAA, para posterior observação ao microscópio de fluorescência, tendo sido empregada a técnica de Martin (1959). A atividade dos vetores de polinização foi monitorada pela observação visual direta no campo. Quando possível, o vôo das abelhas foi acompanhado para a investigação de uma possível discriminação entre as espécies de Diplusodon. Os insetos capturados foram eterizados e mantidos a seco, sendo identificados pelo Dr. Anthony Raw, da Universidade de Brasília.

\section{RESULTADOS}

De acordo com a comparação da morfologia vegetativa e floral das espécies de Diplusodon), foram constatadas semelhanças entre os arbustos ( $D$. oblongus, $D$. ramosissimus e $D$. rosmarinifolius) e entre os subarbustos ( $D$. crulsianus e $D$. villosus). O número de estames (23 em $D$. crulsianus, $17 \mathrm{em} D$. villosus, $16 \mathrm{em} D$. ramosissimus, $15 \mathrm{em} D$. rosmarinifolius e $12 \mathrm{em} D$. oblongus) foi considerado a melhor característica para a diferenciação das espécies. Outra característica relevante encontrada foi a pilosidade que reveste os ramos caulinares e o ovário de $D$. crulsianus e $D$. villosus.

\section{Morfologia floral}

As flores de Diplusodon apresentam-se em panículas, têm antese diurna, são produtoras de pólen, têm corola róseo-forte contrastando com estames amarelos (em pincel) e permanecem receptivas por um dia. Apresentam um certo grau de enantiostilia, que é constatado durante o desabrochar da flor, sendo o estigma o primeiro verticilo reprodutivo que emerge quando as sépalas começam a desabrochar, tornando-se arqueado (para a direirta ou esquerda), de acordo com a inserção da flor na inflorescência (Fig. 1).

\section{Floração}

De acordo com a figura 2, constatam-se dois períodos de floração sincrônica, um de $D$. oblongus e $D$. villosus durante a estação chuvosa (janeiro a abril) e outro de $D$. crulsianus e $D$. ramosissimus e $D$. rosmarinifolius durante a estação seca (maio a agosto). Todas as espécies floresceram por cerca de três meses, exceto D. oblongus em 1991, que floresceu durante 4 meses.

Biologia reprodutiva

De acordo com os dados obtidos, a floração em dois períodos, foi considerada a primeira barreira de intercruzamento para as 5 espécies de Diplusodon. A interpretação dos resultados indica: a) que todas as espécies são capazes de autopolinização e os processos de autogamia se apresentam em baixa taxa de proporção (entre $5 \%$ e $17 \%$ ); b) que a autopolinização espontânea se apresenta naturalmente em todas as espécie (3 a $12 \%$ ) estando bem correlacionada com a autogamia; c) que a polinização natural é 


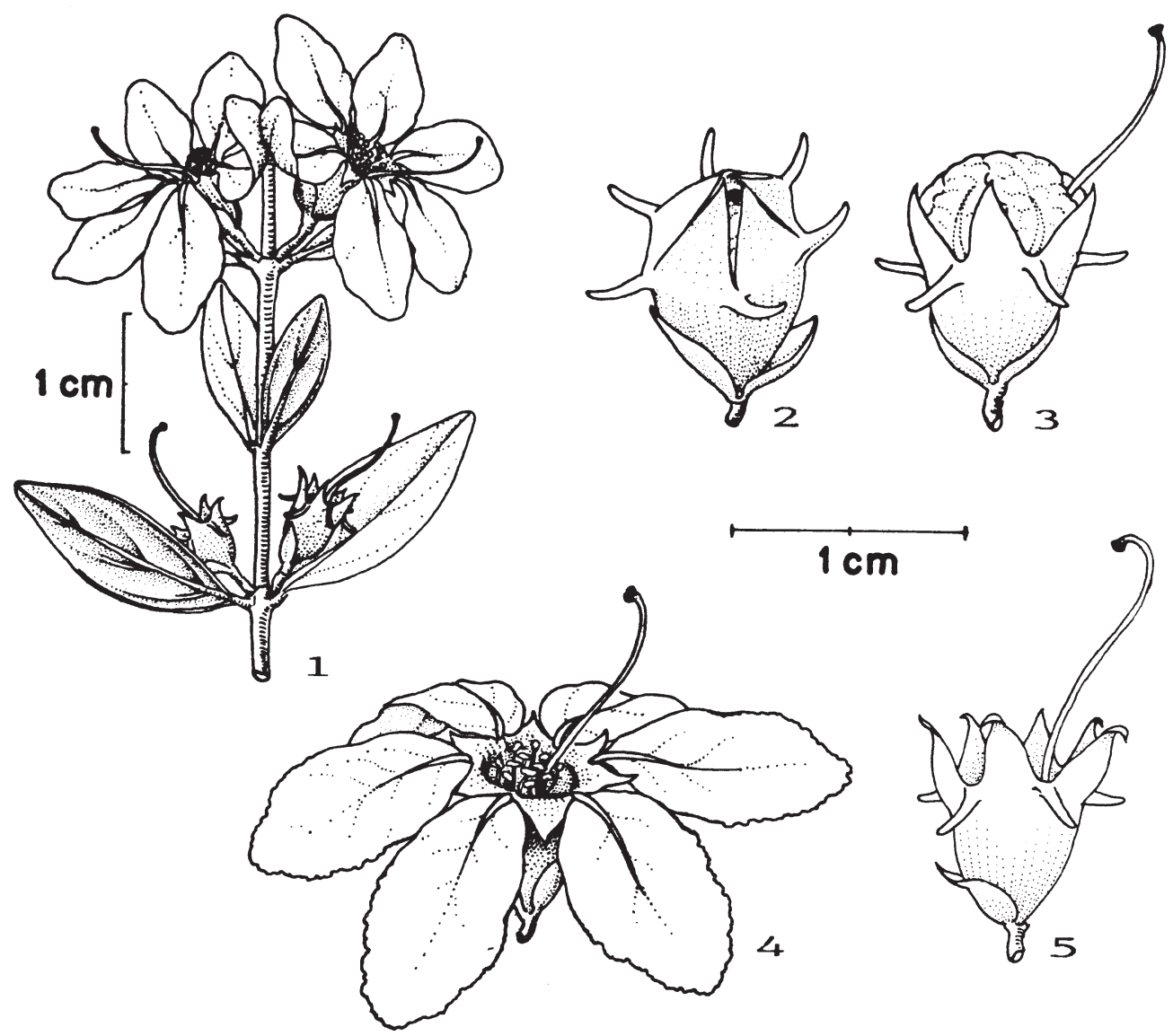

Fig. 1. Diplusodon oblongus Pohl. 1. ramo florido destacando-se a enantiostilia. 2. botão em pré-antese. Notar o estigma surgindo. 3. inicio da antese. Notar estilo arqueado. 4. flor com estigma receptivo. 5. estilo arqueado em flor velha.

sempre muito alta (de 44 a $71 \%$ ), sendo maior na polinização cruzada artificial (66 a $90 \%$ ); d) que dos cruzamentos interespecíficos só foram produzidos híbridos em espécies sincrônicas (cerca de $2 \%$ ); e) que não ocorre apomixia. Os experimentos de polinização (Tabela 1 e Tabela 2), mostraram que as flores de Diplusodon são xenogâmicas, apresentando alta taxa de frutos resultantes de polinização cruzada (de $66 \%$ em $D$. villosus e $90 \%$ em $D$. ramosissimus). As espécies subarbustivas $D$. crulsianus e $D$. villosus 
Granja Barros: Biologia Reprodutiva e Polinização de Espécies Simpátricas de Diplusodon

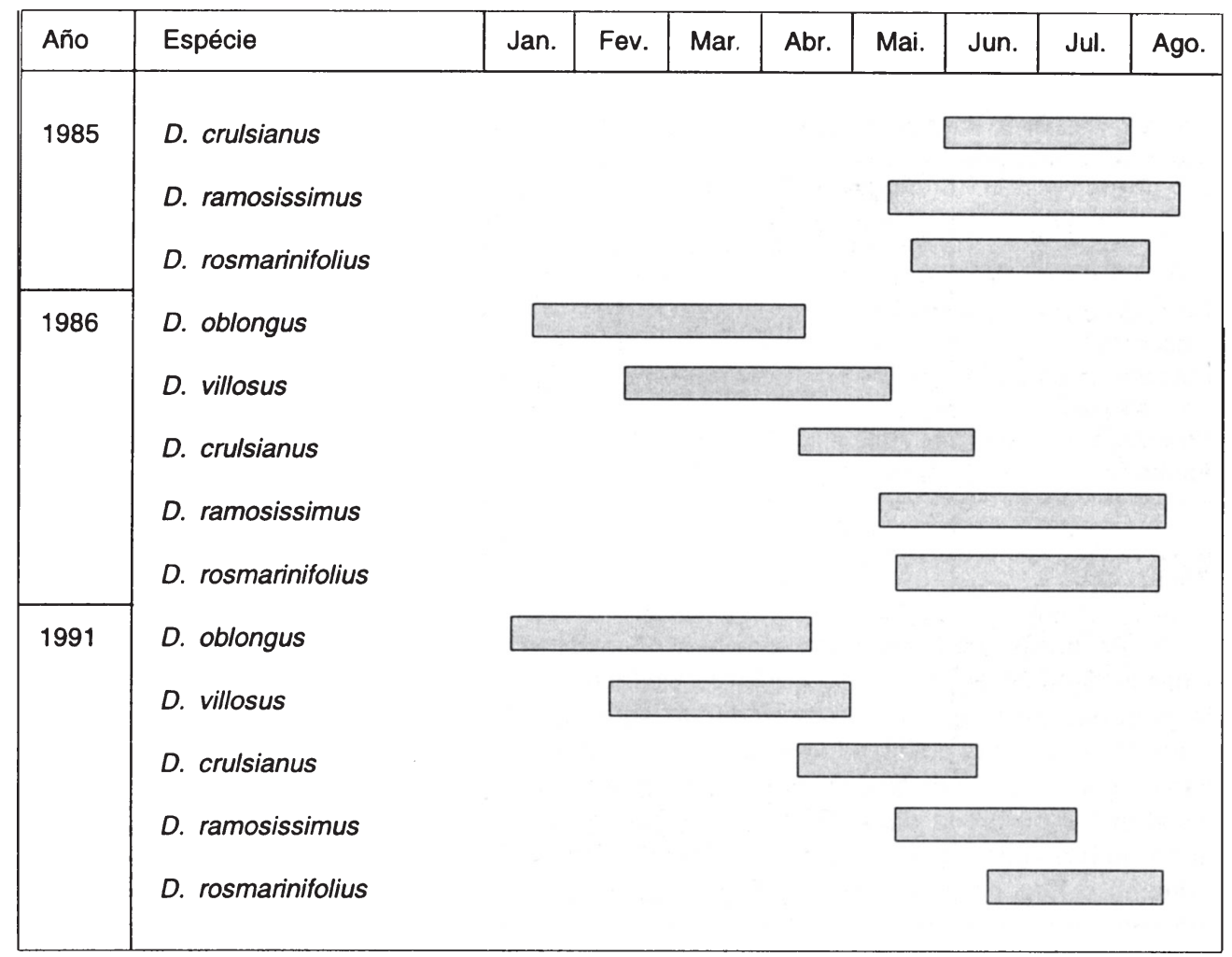

Fig. 2. Comparação do período de floração das espécies de Diplusodon.

demonstraram certo grau de autocompatibilidade com frutos (16\% e $17 \%$ ) resultantes de autopolinização. Os frutos maturados de polinização interespecífica resultaram de espécies com floração sincrônica ( $3 \%$ em $D$. villosus $\times$ D. oblongus e $2 \%$ em $D$. oblongus $\times D$. villosus, $2 \%$ em $D$. villosus $\times D$. crulsianus). Com relção aos cruzamentos interespécificos (pólen armazenado) em espécies de floração assincrônica, é impossível separar o efeito causado pela armazenagem da real incompatibilidade interespecífica que deve existir. Observações de pistilos pós-polinizados ao microscópio de fluorescência revelaram tubos polínicos resultantes de autopolinização em $D$. oblongus e $D$. ramosissimus ( $24 \mathrm{~h}$ e $48 \mathrm{~h}$ ), na base do estilete e no ovário; e em $D$. villosus (24h), foram observados tubos polínicos penetrando na micrópila. Os tubos polínicos resultantes de cruzamentos interespecíficos entre $D$. rosmarinifolius $\times D$. villosus e $D$. oblongus $\times D$. villosus $(48 \mathrm{~h}$ e $72 \mathrm{~h}$ ) foram raramente encontrados no ovário e tinham aparência de malformação. Os tubos polínicos resultantes de auto-polinização espontânea ( $24 \mathrm{~h}$ e $72 \mathrm{~h}$ ) foram comumente detectados nos ovário das espécies subarbustivas e raramente nas arbustivas, porém 
nenhum foi observado alcançando a micrópila. Estudos detalhados serão necessários para o entendimento deste tipo de auto-incompatibilidade de ação tardia.

Tabela 1. Resultado dos testes dos sistemas reprodutivos de Diplusodon ( $n=$ número de flores, $f=$ frutos).

\begin{tabular}{|l|c|c|c|c|c|}
\hline \multirow{2}{*}{ Testes } & $D$. crulsianus & $D$. oblongus & D. ramosissimus & D. rosmarinifolius & D. villosus \\
\cline { 2 - 6 } & $\%(\mathrm{n} / \mathrm{f})$ & $\%(\mathrm{n} / \mathrm{f})$ & $\%(\mathrm{n} / \mathrm{f})$ & $\%(\mathrm{n} / \mathrm{f})$ & $\%(\mathrm{n} / \mathrm{f})$ \\
\hline $\begin{array}{l}\text { Auto polinização } \\
\text { induzida }\end{array}$ & $16(83 / 13)$ & $5(230 / 11)$ & $6(93 / 6)$ & $8(95 / 18)$ & $17(142 / 24)$ \\
$\begin{array}{l}\text { Auto polinização } \\
\text { espontânea }\end{array}$ & $12(64 / 8)$ & $6(180 / 11)$ & $3(100 / 3)$ & $8(100 / 8)$ & $12(65 / 8)$ \\
$\begin{array}{l}\text { Polinização natural } \\
\text { Apoximia }\end{array}$ & $\begin{array}{c}44(150 / 64) \\
-(40 /-)\end{array}$ & $\begin{array}{c}71(150 / 107) \\
-(62 /-)\end{array}$ & $\begin{array}{c}53(141 / 75) \\
-(75 /-)\end{array}$ & $\begin{array}{c}45(132 / 60) \\
-(41 /-)\end{array}$ & $\begin{array}{c}45(73 / 33) \\
-(46 /-)\end{array}$ \\
\hline
\end{tabular}

Polinização

As flores de Diplusodon atraíram 16 espécies de abelhas (Tabela 3), não tendo ocorrido sazonalidade na frequência de visitação durante os períodos de estudo. Abelhas de pequeno porte $(10 \mathrm{~mm})$ como Trigona spinipes, Augochloropsis spp., e Apis mellifera foram consideradas polinizadores efetivas devido à eficiência de polinização e à constância diária de visitação em grupos a úma só planta. As visitas destas 3 espécies de abelhas iniciaram-se por volta das 6:30 h, intensificaram-se de 10:00 h às 12:00 h, decrescendo às 14:00 h e voltando a aumentar das 15:00 h às 16:30 h, quando começaram novamente a diminuir. Trigona visitou as flores de Diplusodon, breve e intensamente (1-2 segundos), enquanto Augochloropsis e Apis as visitaram mais demoradamente (2-5 segundos). Com exceção de Trigona spinipes e Apis mellifera (que é introduzida), as outras abelhas retiram pólen por vibração das anteras. Da investigação do comportamento de Trigona, Augochloropsis e Apis durante o vôo, não foi constatada discriminação entre as espécies de Diplusodon e sim maior visitação às flores de $D$. oblongus. As abelhas menores (5 $\mathrm{mm}$ ), como Trigonisca, Lasioglossum e Pseudoaugochloropsis foram consideradas pilhadoras de pólen, por não terem sido observadas contactando o estigma.

Tabela 2. Resultado dos cruzamentos inter-específicos em Diplusodon ( $n=$ número de flores, $f=$ frutos maturados).

\begin{tabular}{|l|c|c|c|c|c|}
\hline \multirow{1}{*}{ Testes } & D. crulsianus & D. oblongus & D. ramosissimus & D. rosmarinifolius & D. villosus \\
\cline { 2 - 6 } & $\%(\mathrm{n} / \mathrm{f})$ & $\%(\mathrm{n} / \mathrm{f})$ & $\%(\mathrm{n} / \mathrm{f})$ & $\%(\mathrm{n} / \mathrm{f})$ & $\%(\mathrm{n} / \mathrm{f})$ \\
\hline D. crulsianus & $76(87 / 66)$ & $-(50 /-)$ & $-(50 /-)$ & $-(52 /-)$ & $-(59 /-)$ \\
D. oblongus & $-(60 /-)$ & $82(200 / 64)$ & $-(67 /-)$ & $-(48 /-)$ & $2(51 / 1)$ \\
D. ramosissimus & $-(93 /-)$ & $-(85 /-)$ & $90(69 / 62)$ & $-(69 /-)$ & $-(43 /-)$ \\
D. rosmarinifolius & $-(77 /-)$ & $-(81 /-)$ & $-(40 /-)$ & $85(55 / 47)$ & $-(42 /-)$ \\
D. villosus & $2(52 / 1)$ & $3(69 / 2)$ & $-(52 /-)$ & $-(35 /-)$ & $66(65 / 43)$ \\
\hline
\end{tabular}


Granja Barros: Biologia Reprodutiva e Polinização de Espécies Simpátricas de Diplusodon

Tabela 3. Visitantes das flores de Diplusodon durante $1985 / 1986(a=$ abundante, $f=$ frequente, $0=$ ocasional).

\begin{tabular}{|c|c|c|c|c|c|}
\hline \multirow[b]{2}{*}{ Visitantes } & \multicolumn{2}{|c|}{ Período chuvoso } & \multicolumn{3}{|c|}{ Período seco } \\
\hline & D. obl. & D. vil. & D. crul. & D. ramos. & D. rosm. \\
\hline ANTHOPHORIDAE & & & & & \\
\hline Exomalopsis sp. & 0 & - & - & - & 0 \\
\hline $\begin{array}{l}\text { Paratetrapedia (Xanthopedia) } \\
\text { xanthopoda (Moure) }\end{array}$ & $f$ & $f$ & 0 & $f$ & 0 \\
\hline Tetrapedia rugulosa Friese & $f$ & $f$ & 0 & $f$ & $f$ \\
\hline Xylocopa (Neoxylocopa) hirsutissima Maidl. & o & 0 & - & o & - \\
\hline APIDAE & & & & & \\
\hline Apis mellifera L. & a & $f$ & $f$ & a & a \\
\hline Bombus morio (Swederus) & 0 & - & - & - & - \\
\hline Paratrigona lineata (Lepeletier) & $\mathrm{f}$ & 0 & - & 0 & $f$ \\
\hline Plebeia droryana (Friese) & 0 & 0 & 0 & - & - \\
\hline Tetragona clavipes $\mathrm{F}$. & $f$ & $f$ & 0 & 0 & 0 \\
\hline Trigona spinipes $\mathrm{L}$. & a & $f$ & $f$ & $f$ & a \\
\hline Trigonisca sp. & a & $f$ & $f$ & $f$ & $f$ \\
\hline HALICTIDAE & & & & & \\
\hline Augochloropsis aphrodite (Schrottky) & $\mathrm{f}$ & a & $f$ & 0 & $f$ \\
\hline A. cupreola (Cockerell) & $f$ & $f$ & $f$ & 0 & 0 \\
\hline Lasioglossium sp. & $\mathrm{f}$ & $\mathrm{f}$ & a & $\mathrm{f}$ & $f$ \\
\hline Pseudoaugochloropsis graminea $\mathrm{F}$. & f & $f$ & 0 & 0 & $f$ \\
\hline Thectochlora alaris (Vachal) & $f$ & 0 & 0 & 0 & 0 \\
\hline
\end{tabular}

\section{DISCUSSÃO}

As estratégias florais de Diplusodon permitem a classificação das flores no tipo Papaver (Vogel, 1978), que corresponde a flores produtoras de pólen. Considerando o arqueamento e posicionamento lateral dos estilos, discute-se pela primeira vez dentro da família Lythraceae sinais de enantiostilia, fenômeno anteriormente evidenciado em Solanum (Buchmann, 1983; Buchmann et al., 1977), Melastoma, Tibouchina (Dulberger, 1979), Cyanella (Dulberger e Ornduff, 1980), Cassia (Dulberger, 1981) e em Qualea spp. (Barbosa, 1983). Dulberger (1979) associa enantiostilia a flores produtoras de pólen, anteras poricidas, pólen retirado por vibração das anteras "buzz pollination" e estigma diminuto. Com exceção das anteras (que têm deiscência longitudinal), as flores de Diplusodon se enquandram nestas categorias. A função da enantiostilia nas flores de Diplusodon é associada à proteção dos estiletes contra o quebramento, pois este é deslocado quando da retirada do pólen, e como uma estratégia floral para minimizar a contaminação com o pólen da mesma flor apesar de não impedir a geitonogamia. Dulberger e Ornduff (1980) e Dulberger (1981) apontaram enantiostilia como um fator de promoção da polinização cruzada em Cyanella, Cassia auriculata e C. didymobotria, cujas flores 
apresentam simetria floral dimórfica. Aparentemente, este fenômeno pode ser sugerido para Diplusodon, que desabrocha simultâneamente duas flores (uma com o estilo voltado para a esquerda e outra com estilo voltado para a direita) na mesma inflorescência, simulando uma certa dimorfia floral.

A similaridade floral, a sincronia de floração e a simpatria nas espécies de Diplusodon favorecem o fluxo indiscriminado de polinizadores devido à manutenção da fonte alimentar, como sugerido por Barros (1989), para espécies de Tabebuia, Erythroxylum e Kielmeyera. A estrutura floral de Diplusodon com estames em pincel, certamente permite a Augochloropsis e outras abelhas nativas a possibilidade da retirda do pólen por vibração das anteras. Até o presente, este tipo de comportamento de abelhas tem sido frequentemente citado para as flores com anteras poricidas (Laroca, 1970; Bowers, 1975; Dulberger, 1979, 1981; Camargo et al., 1984; Frankie et al., 1990). Em espécies do cerrado "buzz pollination" foi evidenciada também em flores com estames em pincel e anteras rimosas como em Kielmeyera coriacea, K. speciosa (Barros, 1989; Oliveira e Sazima, 1990), K. abdita, K. variabilis (Barros, 1989), e em 4 espécies de Myrtaceae (Proença, 1992). Bittrich et al. (1993), também constataram este fenômeno em flores de Ternstroemia laevigata e $T$. dentata (Theaceae), cujos estames são numerosos e em forma de pincel.

A floração de Diplusodon em dois períodos (seco e chuvoso) sugere uma partilha temporal do recurso polinizador, como observado em Kielmeyera (Barros, 1989; Oliveira e Sazima, 1990), Byrsonima (Barros, 1992) e em 2 espécies de Bombacaceae (Fischer et al., 1992). O padrão de florescimento das espécies arbustivas ( $D$. oblongus, $D$. ramosissimus e $D$. rosmarinifolius) pode ser associado ao "cornucopian type" e das subarbustivas ( $D$. crulsianus e $D$. villosus) ao "steady state pattern" sugeridos por Gentry (1974) para Bignoniáceas tropicais. No primeiro caso, desabrocham muitas flores por vez e no segundo, poucas; em ambos padrões, durante várias semanas. Quanto à convergência de floração de $D$. oblongus/ $D$. villosus e $D$. crulsianus/ $D$. ramosissimus/ $D$. rosmarinifolius, sugere-se algum tipo de mutualismo floral, resultando em um incremento de recompensas, como meio de promover a polinização cruzada. Embora tal convergência possa implicar na possibilidade de polinização interespecífica, este parece ser um padrão observado em outros casos de relação planta/polinizador (Schemske, 1981; Feinsinger, 1983). Sincronia de floração foi investigada tambem em espécies de Heliconia (Stiles, 1975), Costus (Schemske, 1981), Dalechampia (Armbruster e Herzig, 1984) Byrsonima (Barros, 1992), e em estudos sobre comunidades vegetais (Wheelwright, 1985; Murray et al., 1987) que demonstraram maior convergência de floração que o esperado, e que as curvas de florescimento das diferentes espécies se apresentavam mais fortemente agregadas que distribuídas dentre as espécies congenéricas.

O sucesso reprodutivo da polinização artificial cruzada em Diplusodon, quando comparada à polinização natural (controle), é entendido como consequência de polinização inadequada com pólen autógamo/geitonógamo depositado por agentes naturais. Paralelamente, em vários táxons de cerrado: Qualea (Barbosa, 1983), Caryocar (Gribel, 1986), Acrocomia (Scariot, 1987), Styrax (Saraiva et al., 1988), Tabebuia, Kielmeyera e Erythroxylum (Barros, 1989), Jacaranda (Vieira et al., 1992) Aspidosperma, Vellozia, Eriotheca (Oliveira, 1991), Campomanesia, Eugenia, Myrcia (Proença, 1991), também foi documentada maior produção de frutos de polinização cruzada, que de controle. A autocompatibilidade parcial das espécies subarbustivas $D$. crulsianus e $D$. villosus sugere 
estratégias evolutivas para minimizar a competição por poliniadores entre co-ocorrentes intragenéricos arbustivos. Isto também foi evidenciado em Erythroxylum campestre, que é simpátrica com E. tortuosum e E. suberosum, ambas arbóreo-arbustivas (Barros, 1989). Autocompatibilidade tem sido referenciada em espécies herbáceas, arbustivas e trepadeiras como Ipomoea hederifolia e I. quamoclit (Machado e Sazima, 1987), Angelonia pubescens (Vogel e Machado, 1991), Campomanesia pubescens (Proença, 1991), Pyrostegia venusta (Gobatto-Rodrigues e Stort, 1992), que sofrem flutuações anuais nas populações, devido à sazonalidade ambiental.

\section{LITERATURA CITADA}

Armbruster, W. S. e A. L. Herzig. 1984. Partioning and sharing of pollinators by four simpatric species of Dalechampia (Euphorbiaceae) in Panama. Ann. Missorui Bot. Gard. 71: 1-6.

Barbosa, A. A. 1983. Aspecto da ecologia reprodutiva de Qualea (Vochysiaceae) num cerrado de Brasília, DF, Brasil. Dissertação de Mestrado. Universidade de Brasília. Brasília. 91 pp.

Barros, M. A. G. 1989. Studies on the pollination biology and breeding systems of some genera, with sympatric species in the Brazilian cerrados. Ph. D. Thesis. University of St. Andrews. Scotland. $246 \mathrm{pp}$.

Barros, M. A. G. 1992. Fenologia de floração, estratégias reprodutivas e polinização de espécies simpátricas do gênero Byrsonima Rich. (Malpighiaceae). Revista Brasil. Biol. 52: 343-353.

Bittrich, V., M. C. E. Amaral e G. A. R. Melo. 1993. Pollination biology of Ternstroemia laevigata Wawra and T. dentata (Theaceae). PI. Syst. Evol. 185: 1-6.

Bowers, K. W. A. 1975. The pollination ecology of Solanum rostratum (Solanaceae). Am. J. Bot. 62: 633638.

Buchmann, S. L. 1983. Buzz pollination in Angiosperms. In: Jones, C. E. e J. Little (eds.). Handbook of experimental pollination biology. Scientific and Academic Ed. New York. pp. 73-113.

Buchmann, S. L., C. E. Jones e J. Colin. 1977. Vibratile pollination of Solanum douglassii and S. xanti (Solanaceae) in Southern California. Wasmann J. Biol. 5: 1-25.

Camargo, J. M. F., G. Gottsberger e I. Silberbauer-Gottsberger. 1984. On the phenology and flower visiting behaviour of Oxaea flavescens (Klug) (Oxaeinae, Andreidae, Hymenoptera) in São Paulo, Brazil. Beitr. Biol. Pflanzen 59: 159-179.

Cavalcanti, T. B. 1995. Revisão do gênero Diplusodon Pohl. (Lythraceae). Tese de doutorado. Universidade de São. Paulo. Universidade de São Paulo. São Paulo. 505 pp.

Dulberger, R. 1979. The pollination syndrome of some nectaries flowers visited by vibrating insects. In: Reproduction in flowering plants. Abstract International Symposium Christchurch. New Zealand. 75-77 pp.

Dulberger, R. 1981. The floral biology of Cassia didymobotrya and C. auriculata (Caesalpinoideae). Am. J. Bot. 68: 1350-1360.

Dulberger, R. e R. Ornduff. 1980. Floral morphology and reproductive biology of Cyanella (Tecophilaceae). New Phytol. 86: 45-56.

Feinsinger, P. 1983. Variable nectar secretion in a Heliconia species pollinated by mit hummingbirds. Biotropica. 15: 48-52.

Fischer, E. A., F. A. Jimenez e M. Sazima. 1992. Polinização por morcegos em duas espécies de Bombacaceae na Estação Ecológica da Juréia, São Paulo. Revista Brasil. Bot. 15: 67-72.

Frankie, G., S. B. Winson, L. E. Newstrom, J. F. Barthell, W. A. Habber e J. K. Frankie. 1990. Plant phenology, pollination ecology, pollinator behavior and conservation of pollinators in neotropical dry forest. In: Bawa S., K. S. e M. Hadley. (eds.). Reproductive ecology of tropical forest plants. Parthernon. Carnforth. 37-47 pp. 
Gentry, A. H. 1974. Coevolutionary patterns in Central American Bignoniaceae. Ann. Missouri Bot. Gard. 61: 728-759.

Gobatto-Rodrigues, A. A. e M. N. S. Stort. 1992. Biologia floral e reprodução de Pyrostegia venusta (Ker-Grawl) Miers. Bignoniaceae. Revista Brasil. Bot. 15: 37-41.

Graham, A., J. Nowicke, J. J. Sdvarla, S. A. Graham, V. Patel e S. Lee. 1985. Palinology and systematics of the Lythraceae. Introduction and genera Adenaria through Ginoria. Am. J. Bot. 72: 1012-1031.

Gribel, R. 1986. Ecologia da polinização e da dispersão de Caryocar brasiliense Camb. (Caryocaraceae) na Região do Distrito Federal. Dissertação de Mestrado. Universidade de Brasília. Brasília. 143 $\mathrm{pp}$.

Heringer, E. P., G. M. Barroso, J. A. Rizzo e C. T. Rizzini. 1977. A flora do cerrado. In: Ferri, M. G. (ed.) IV Simpósio sobre o cerrado. Editora Universidade de São Paulo. São Paulo. pp. 211232.

Laroca, S. 1970. Contribuição para o conhecimento das relações entre as abelhas e flores. Coleta de pólen das anteras tubulares de certas Melastomatáceas. Revista Florestal 2: 69-74.

Lourteig, A. 1989. Lythraceae austroamericanae. Addenda et corrigenda II. Bradea 5: 205-242.

Machado, I. C. S. e Sazima, M. 1987. Estudo comparativo em duas espécies invasoras: Ipomoea hederifolia e I. quamoclit (Convolvulaceae). Revista Brasil. Biol. 47: 425-436.

Martin, F. W. 1959 - Staining and observing pollen tubes in the style by means of fluorescence. Stain Technol. 34: 125-128.

Murray, K. G., P. Feinsinger, W. H. Busby, Y. B. Linhart, J. H. Beach e S. Kinsman. 1987. Evaluation on character displacement among plants in two tropical pollination guilds. Ecology 68: 1283-1293.

Oliveira, P. E. A. 1991. The pollination and reproductive biology of a cerrado woody community in Brazil. Ph. D. Thesis. University of St. Andrews. Scotland. $138 \mathrm{pp}$.

Oliveira, P. E. A. e M. Sazima. 1990. Pollination biology of two species of Kielmeyera (Guttiferae) from Brazilian cerrado vegetation. PI. Syst. Evol. 172: 35-49.

Oliveira, R. C. 1991. Ciclo reprodutivo de Diplusodon orbicularis Koehne (Lythraceae). Dissertação de Mestrado. Universidade de São Paulo. São Paulo. 118 pp.

Proença, C. E. B. 1991. The reproductive biology and taxonomy of the Myrtaceae of the Distrito Federal (Brazil). Ph. D. Thesis. University of St. Andrews. Scotland. $278 \mathrm{pp}$.

Proença, C. E. B. 1992. Buzz pollination - older and more widespread than we think ? J. Trop. Ecol. 8: $115-120$.

Ratter, J. A. 1980. Notes on the vegetation of the Fazenda Água Limpa (Brasilia. Distrito Federal. Brazil) including a key to the genera of dicotyledons of cerrado. Royal Botanic Garden. Edinburgh.

Saraiva, L. C., O. Cesar y R. Monteiro. 1988. Biologia da polinização e sistema de reprodução de Styrax camporum Pohl e S. ferrugineus Nees et Mart. (Styracaceae). Revista Brasil. Bot. 11: $71-80$.

Scariot, A. O. 1987. Biologia reprodutiva de Acrocomia aculeata (Jacquin) Loddies ex Martius (Palmae) na região do Distrito Federal. Dissertação de Mestrado. Universidade de Brasilia, Brasília. 108 $\mathrm{pp}$.

Schemske, D. 1981. Floral convergence and pollinator sharing in two bee-pollinated tropical herbs. Ecology 62: 946-954.

Stiles, F. G. 1975. Ecology, flowering phenology and hummingbird pollination of some Costa Rican Heliconia species. Ecology 56: 285-301.

Vieira, M., R. M. S. A. Meira, L. P. Queiroz e J. A. A. Meira Neto. 1992. Polinização e reprodução de Jacaranda caroba (Vell.) D. C. (Bignoniaceae) em área de cerrado do sudoeste brasileiro. Anais do VIII Congresso da Sociedade Botânica de São Paulo. São Paulo. pp. 13-19.

Vogel, S. 1978. Evolutionary shifts from reward to deception in pollen flowers. In: Richards, A. (ed.). The pollination of flowers by insects. London. Linn. Soc. Symposium, Series 6. Academic Press. London. 
Granja Barros: Biologia Reprodutiva e Polinização de Espécies Simpátricas de Diplusodon

Vogel, S. e I. C. S. Machado. 1991. Pollination of four sympatric species of Angelonia (Scrophulariaceae) by oil-collecting bees of NE Brazil. PI. Syst. Evol. 178: 153-178.

Wheelwright, N. 1985. Competition for dispersers and the timing for flower and fruiting in a guild of tropical trees. Oikos 44: 465-477. 